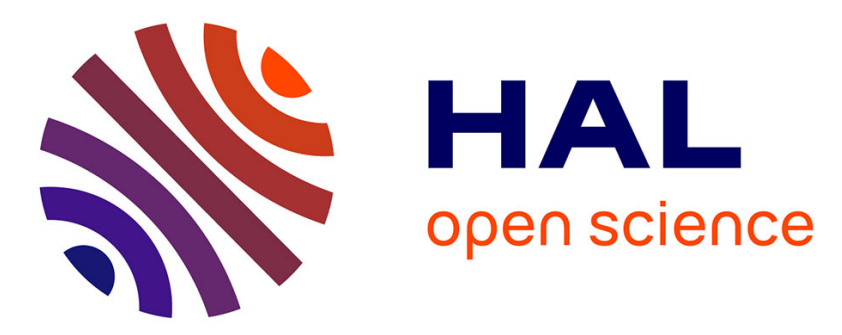

\title{
Geometric analysis of the singularities of a magnetic manipulation system with several mobile coils.
}

\author{
Baptiste Veron, Arnaud Hubert, Joël Abadie, Nicolas Andreff
}

\section{To cite this version:}

Baptiste Veron, Arnaud Hubert, Joël Abadie, Nicolas Andreff. Geometric analysis of the singularities of a magnetic manipulation system with several mobile coils.. IEEE/RSJ International Conference on Intelligent Robots and Systems, IROS'13., Jan 2013, Japan. pp.4996-5001. hal-00903623

\section{HAL Id: hal-00903623 \\ https://hal.science/hal-00903623}

Submitted on 12 Nov 2013

HAL is a multi-disciplinary open access archive for the deposit and dissemination of scientific research documents, whether they are published or not. The documents may come from teaching and research institutions in France or abroad, or from public or private research centers.
L'archive ouverte pluridisciplinaire HAL, est destinée au dépôt et à la diffusion de documents scientifiques de niveau recherche, publiés ou non, émanant des établissements d'enseignement et de recherche français ou étrangers, des laboratoires publics ou privés. 


\title{
Geometric analysis of the singularities of a magnetic manipulation system with several mobile coils.
}

\author{
Baptiste Véron, Arnaud Hubert, Joël Abadie and Nicolas Andreff
}

\begin{abstract}
It has been demonstrated that magnetic fields are relevant for manipulating an untethered magnet, either using fixed coils or mobile permanent magnets. This paper shows however, that any magnetic manipulation method is prone to singular configurations and that the simple numerical analysis of the rank of the "manipulation matrix" is not enough to detect them. Alternatively, we propose a geometrical analysis to interpret and detect the singularities as well as to decide on the acceptability of a reference trajectory. Then, we present results obtained by simulating a planar manipulation system including a multiple mobile coils and a Helmholtz like set-up.
\end{abstract}

\section{INTRODUCTION}

This work is in the context of the gastrointestinal endoscopy. Since the development of the M2A capsule endoscopy system [1], several studies have been led to improve the concept of capsular device navigating through the gastrointestinal tracts, especially about the two challenges of the localization and the controlled manipulation of the capsule. This paper focuses on this second challenge: replacing passive locomotion (peristaltis) of the capsule, by an active control of the motion.

One way to control the capsule motion is to add legs to the capsule [2], but this yields strong energy concerns.

An alternative to embedded actuation is to actuate the capsule externally using magnetic fields. This presents the advantage of being non toxic to the human body [3] and it permits to remotely control the device without any potentially traumatic gripping in the intestinal wall (due to the legs). Two approaches can be found in the literature for this type of magnetic manipulation.

The first method employs stationary electromagnets and then control their currents to adjust the magnetic field (as in opthalmology) [4], [5]. In a Helmholtz configuration, the workspace lies between the two coils where a uniform magnetic field is present [6]. This is used in MRI systems to displace a magnetic micro-object within the blood vessels [7], [8].

The second method makes use of permanent magnets to create the appropriate magnetic field and they are moved around the patient using a robotic system to displace the manipulated object [9], [10]. On some of these systems, the "driving permanent magnet" is constantly rotating [11], [12].

Existing work (Table I) shows that the two approaches are complementary.

The stationary electromagnets approach has full dexterity. However the electrical consumption (associated to heat

The authors are with the FEMTO-ST Institute - UMR CNRS 6174 - AS2M department (Automatic control and Micro-Mechatronic Systems) - 24 rue Alain Savary, 25000 Besancon (FRANCE).

\begin{tabular}{|l|l|l|}
\hline Static coils & Mobile magnets & Mobile coils \\
\hline \hline$\oplus$ Full dexterity & $\ominus$ Partial dexterity & $\oplus$ Full dexterity \\
$\ominus$ Long source-to- & $\oplus$ Short source-to- & $\oplus$ Short source-to- \\
object distance & object distance & object distance \\
$\ominus$ Heat & $\oplus$ Thermally inert & $\rightsquigarrow$ Reduced heat \\
$\oplus$ On/off capacity & $\ominus$ Always "on" & $\oplus$ On/off capacity \\
$\oplus$ Stabilizable & $\ominus$ Intrinsic unstability & $\oplus$ Stabilizable \\
$\oplus$ Simple control & $\oplus$ Simple control & $\oplus$ Redundant control \\
$\ominus$ Poor patient ac- & $\ominus$ Dangerous to the & $\rightsquigarrow$ Light weight sys- \\
ceptability & patient and staff & tem \\
\hline
\end{tabular}

TABLE I: Some pros and cons of magnetic manipulation techniques (from [13])

concerns) becomes high as the capsule evades from the sources. Conversely, the mobile magnets approach does not need electrical energy to generate the field, but the use of a constant field is intrinsically unstable (since the capsule is always attracted by the magnet, which can not be "turned off") and offers only partial dexterity (because the continuity of motion is much more restrictive, with respect to time, than the continuity of the magnetic flux).

Our concept, depicted in Fig. 1, consists of several coils actuated by a robotic system so that they can move around the patient and stay close to the capsule. Hence, it keeps full dexterity by making use of coils while reducing energy consumption by a nearer manipulation. Moreover, the robotic system, yet to be properly designed, can be made lighter and less bulky than the existing prototypes, in order to be intrinsically safe to the patient.

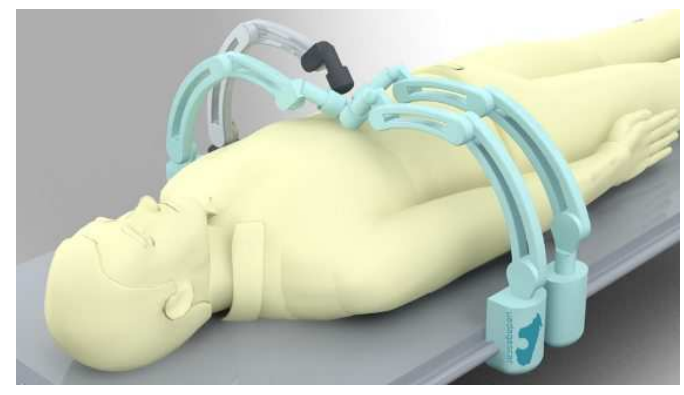

Fig. 1: Robot-assisted magnetic manipulation with several mobile coils.

Recently, we proposed in [13], [14] an hybrid approach by using multiple mobile coils in order to control the motion of a magnetic capsule by both servoing the currents and moving the magnetic sources. Modification of the magnetic field then becomes possible mechanically and electrically. A redundant system is therefore created which increases manipulability and dexterity. To verify this ability we study the control of such system. 
However, the complexity of this system is prone to singularities [14]. This paper contributes to show that the usual condition number is not enough for a reliable detection of such singularities and to propose, as an additional criterion, the use of the angles between the columns of the "manipulation matrix" which models the current-to-force conversion. These angles are used to determine the acceptability of a reference trajectory, which is shown by simulating a multi mobile coils system and a Helmholtz like set-up.

This paper is organized as follows. Section II presents the theoretical background for magnetic manipulation and singularity analysis, while Section III focuses on the study of a particular case. Then Section IV presents the application of this theory by simulating two planar manipulation systems. Conclusion and perspectives are given in Section V.

\section{A HYBRID APPROACH TO MAGNETIC MANIPULATION}

\section{A. Basic electromagnetics seen from a robotics viewpoint}

The motion of the capsule is driven by the efforts applied on it. These efforts are due to the magnetic field created by each coil (the magnetic force $\mathbf{F}_{\mathbf{m}, \mathbf{i}}$ and torque $\mathbf{C}_{\mathbf{m}, \mathbf{i}}$ ), the weight of the capsule $(m \mathbf{g})$, and friction force $\mathbf{F}_{\mathbf{f}}$ and torque $\mathbf{C}_{\mathbf{f}}$. All these efforts and accelerations are expressed in $\mathscr{F}_{0}$, a fixed reference frame which can be attached to the patient, to the perception system, or to the patient's bed. $\mathscr{F}_{o b j}$ is the local frame linked to the moving object. The notation ${ }^{i} \mathbf{X}$ used in the rest of the paper points out in which reference frame $\mathscr{F}_{i}$ the vector $\mathbf{X}$ is expressed. If nothing is specified, the vector is expressed in $\mathscr{F}_{0}$.

The magnetic efforts applied on a permanent magnet located at the point $\mathbf{P}$ with a magnetization vector $\mathbf{M}$ (which encompasses the magnet strength and its orientation in space) in an external magnetic field $\mathbf{B}_{\mathbf{i}}(\mathbf{P})$ created by a coil $i$, are given by [15]:

$$
\begin{aligned}
& \mathbf{F}_{\mathbf{m}, \mathbf{i}}=V \cdot \nabla\left(\mathbf{M} \cdot \mathbf{B}_{\mathbf{i}}(\mathbf{P})\right) \\
& \mathbf{C}_{\mathbf{m}, \mathbf{i}}=V \cdot \mathbf{M} \wedge \mathbf{B}_{\mathbf{i}}(P)
\end{aligned}
$$

where $V$ is the volume of the permanent magnet, $\nabla$ is the gradient operator and $\wedge$ is the cross-product.

As electromagnets are used, the magnetic field created by the $i$ th coil also depends on the current $I_{i}$ flowing through this coil. Then $\mathbf{B}_{\mathbf{i}}(\mathbf{P})=\mathbf{B}_{\mathbf{i}}\left(\mathbf{P}, I_{i}\right)$. Moreover, computing the magnetic field due to a coil $i$ is usually easier in the frame $\mathscr{F}_{i}$ attached to this coil, but this formula can also be easily expressed in the reference frame $\mathscr{F}_{0}$ using the homogeneous matrix ${ }^{0}[T]_{i}$ multiplication, composed of the orientation and position of $\mathscr{F}_{i}$ with respect to $\mathscr{F}_{0}$ :

$$
\mathbf{B}_{\mathbf{i}}\left(\mathbf{P}, I_{i}\right)={ }^{0}[T]_{i}{ }^{i} \mathbf{B}_{\mathbf{i}}\left({ }^{i} \mathbf{P}, I_{i}\right)
$$

In vacuum, air, or any linear magnetic medium, the superposition principle is used to compute the overall magnetic field created by the $n$ coils. As shown in [5], in such a case, forces and torques can be computed as a linear combination of the currents in the coils, grouped in the vector I. For a system with $n$ coils, the direct electro-magnetic model is then given by:

$$
[A(\mathbf{P}, \mathbf{M})] \mathbf{I}=\left(\begin{array}{l}
\mathbf{F}_{\mathbf{m}} \\
\mathbf{C}_{\mathbf{m}}
\end{array}\right)
$$

By construction, the electro-magnetic manipulation matrix, or simply the "manipulation matrix", $[A]$ depends on: the position of the capsule $\mathbf{P}$, the orientation of the capsule (which can be deduce from $\mathbf{M}$ ), and the position of each coil ${ }^{0}[T]_{i}$.

Moreover, $[A]$ depends non-linearly on $\mathbf{P}$ and $\mathbf{I}$ which does not guarantee that even for simple set-ups (e.g. Helmholtzlike configuration), it will never be singular.

Finally, the three possibilities to control the efforts applied to the capsule appear in this matrix modelling. The first control method is to change the coils position which will modify the matrix $[A]$, the second method is to change the currents in the coils, and the third one is the hybrid one we proposed.

Notice also that $[A]$ can be decomposed as:

$$
[A]=\left[\begin{array}{l}
A_{F} \\
A_{C}
\end{array}\right]
$$

$\left[A_{F}\right]$ and $\left[A_{C}\right]$ are two $3 \times n$ matrices, and the product of these matrices by the vector of currents $\mathbf{I}$ gives respectively the magnetic force $\mathbf{F}_{\mathbf{m}}$ and the magnetic torque $\mathbf{C}_{\mathbf{m}}$.

\section{B. Study of the singularities}

The matrix $[A]$ is a $6 \times n$ matrix, with $n$ the number of coils, i.e. the number of independant currents. The $i$ th column of this matrix represents the wrench on the capsule (force and torque) per current unit created by the $i$ th coil, according to (1) and (2). Thus, $[A]$ has the same structure as a robot kinematic Jacobian matrix. The analysis of its singularities is thus needed.

Numerically, singularities are detected by computing the rank of the matrix (usually, by detecting the fall of the ratio of successive singular values). This is actually related to the condition number of the matrix:

$$
\operatorname{Cond}([A])=\frac{\sigma_{1}}{\sigma_{N}}
$$

with $\sigma_{1} \geqslant \sigma_{2} \geqslant \cdots \geqslant \sigma_{N}>0$, the singular values of $[A]$ obtained by singular value decomposition (SVD).

The condition number is frequently used as a so-called manipulability index [16] to measure the performance of a mechanism. However, in addition to the specificities of the special euclidean group $S E(3)$, this index suffers from the fact that one needs an empirical choice of the threshold value to decide the closeness of the rank deficiency. Unfortunately, this threshold on the ratios of two successive singular values does not have a physical interpretation.

On the opposite, for such kinematic matrices, whose dimensions are $6 \times n$, one can express the singularities from geometry. A geometrical analysis relies on the fact that if $[A]$ is non-singular, a subset of columns which spans the whole space (ie. of dimension 6) exists.

In practice, the relative orientation of each column to each other has a great importance. Indeed, if all these columns are 
almost collinear, without $[A]$ being singular, the necessary energy to move in a "transverse" direction (see Fig. 2) is much higher than in an ideal case (a subset of the columns forming an orthogonal base).

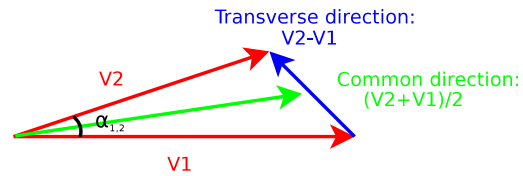

Fig. 2: "Transverse" direction.

To enlighten this point, let us consider a planar manipulator. In this case, the dimension of $[A]$ reduces to $3 \times n$, with the first two rows of $\left[A_{F}\right]$ dedicated to forces in the plane, and the third row of $\left[A_{C}\right]$ to the torque around the normal to the plane. As the direction of the columns of $\left[A_{C}\right]$ is constant, the geometrical analysis can be limited to $\left[A_{F}\right]$. Of course, a singular configuration occurs when the columns of $\left[A_{F}\right]$ are parallel (i.e. $\alpha_{i, j}=0$ ), but a non-singular case with small angles (let us say 5 or $10 \mathrm{deg}$ ) between the columns of $\left[A_{F}\right]$ may also cause significant problems in practice. At right angles, the manipulability is good, because the energy transfer is decoupled. At small angles, it is poor, as more energy is needed to move the capsule in the "transverse" direction (Fig. 2) than in the "common" direction.

This is particularly prevalent in magnetic manipulation, because at small angles, one would need higher currents in the coils than at angles almost equal to $\pi / 2 \mathrm{rad}$. Since "higher currents" means a risk of current saturation as well as heating in the coils, this situation should be avoided. As a consequence, the analysis of the evolution of the angles $\alpha_{i, j}$ (between the $i$ th and the $j$ th column vectors of the matrix $\left.\left[A_{F}\right]\right)$ can be used to check if the trajectory is acceptable, i.e. the trajectory does not encounter a singularity of $[A]$.

\section{STUDY OF A PARTICULAR CASE}

\section{A. Description}

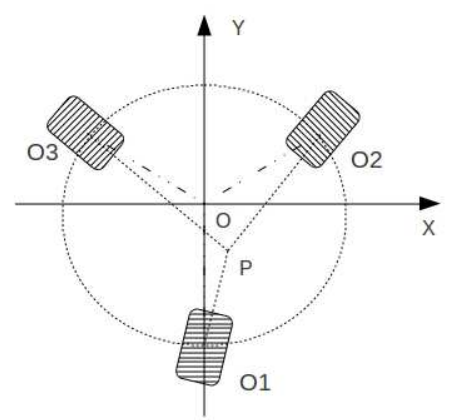

Fig. 3: System studied.

The proposed criteria is investigated in the horizontal planar configuration. Thus, the study is carried on a system (presented in [14]) with $n=3$ coils, and the capsule can move in the plane made by the coils axis as shown on Fig. 3.
The architecture shown is similar to a 3-UPS parallel kinematic manipulator. The coils can turn around a vertical axis $\left(O_{i}, \mathbf{Z}\right)$ in order to keep the mobile on their axis of revolution. This architecture was chosen to be able to use the well-known magnetic dipole approximation (6) often used in literature to compute the magnetic field ${ }^{i} \mathbf{B}_{\mathbf{i}}\left({ }^{i} \mathbf{P}, I_{i}\right)$. This approximation is only valid when the distance between $\mathbf{P}$ and the coil axis is small.

$$
{ }^{i} \mathbf{B}_{\mathbf{i}}\left({ }^{i} \mathbf{P}, I_{i}\right)=\left(\begin{array}{rl}
B_{r} & =\frac{\mu_{0}}{2 \pi}\left(I_{i} \cdot \pi a^{2}\right) \frac{\cos \theta}{r^{3}} \\
B_{\theta} & =\frac{\mu_{0}}{4 \pi}\left(I_{i} \cdot \pi a^{2}\right) \frac{\sin \theta}{r^{3}} \\
B_{\varphi} & =0
\end{array}\right)
$$

\section{B. Modeling}

As the system is studied in the horizontal plane, $\mathbf{P}, \mathbf{M}$ and $\mathbf{B}_{\mathbf{i}}(i=1,2,3)$ are coplanar. Thus, the generic form of the matrix $[A]$ becomes:

$$
[A]=\left[\begin{array}{ccc}
F_{1 x} & F_{2 x} & F_{3 x} \\
F_{1 y} & F_{2 y} & F_{3 y} \\
0 & 0 & 0 \\
0 & 0 & 0 \\
0 & 0 & 0 \\
C_{1 z} & C_{2 z} & C_{3 z}
\end{array}\right]
$$

which can be reduced, as previously mentioned, by suppressing the rows of 0 .

The geometrical analysis is then reduced to the study of the angles $\alpha_{i, j}$ between 2D column vectors of $\left[A_{F}\right]$ (the upper two rows of $[A]$ ).

\section{Simulation Results}

\section{A. Simulation set-up}

A simulation in $\mathrm{C}++$ using the ViSP library [17] has been developed. The trajectory control, based on the structure shown in Fig. 4, is computed. With this control strategy, the orientation control and the current control are independent.

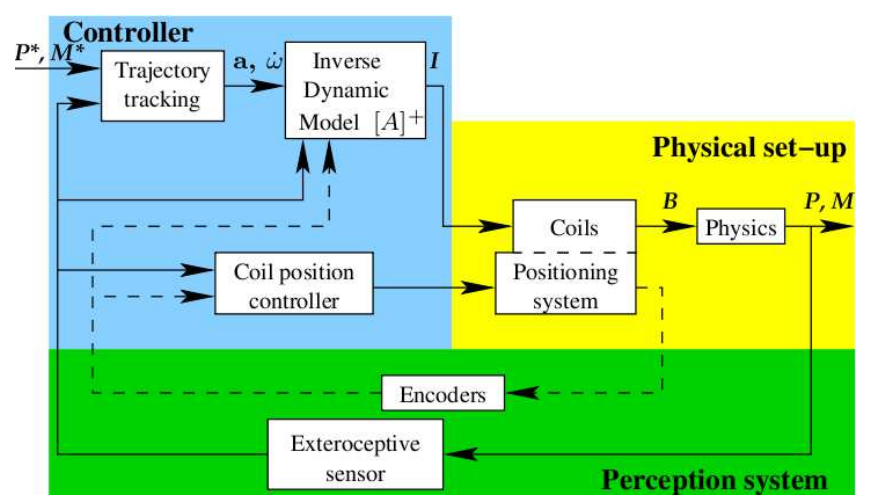

Fig. 4: Control strategy inspired by Parallel Kinematic Machine (PKM) with linear actuators.

The trajectory tracking block is a PD plus feed-forward controller that provides the desired acceleration $\left(\mathbf{a}^{*}, \dot{\omega}^{*}\right)$ for the capsule from the difference between its measured $(\mathbf{P}, \mathbf{M})$ and reference position $\left(\mathbf{P}^{*}, \mathbf{M}^{*}\right)$. To determine the current $\mathbf{I}$ 
needed to ensure $\left(\mathbf{a}^{*}, \dot{\omega}^{*}\right)$, the dynamic model of the capsule is used:

$$
[A(\mathbf{P}, \mathbf{M})] \mathbf{I}=\left(\begin{array}{c}
m \cdot \mathbf{a} \\
{ }^{0}\left[I_{G}\right]_{o b j} \cdot \dot{\omega}
\end{array}\right)-\left(\begin{array}{c}
\mathbf{F}_{\mathbf{f}} \\
\mathbf{C}_{\mathbf{f}}
\end{array}\right)
$$

where $m$ is the mass of the capsule, ${ }^{0}\left[I_{G}\right]_{o b j}$ its inertia, $\mathbf{F}_{\mathbf{f}}$ and $\mathbf{C}_{\mathbf{f}}$ the friction efforts, and $\mathbf{a}$ and $\dot{\omega}$ the acceleration.

This model is inverted by singular value decomposition to calculate the current $\mathbf{I}$ applied into the coils. It is implemented in the block called Inverse Dynamic Model in Fig. 4.

\section{B. Results}

First, the simulation is ran without any friction $\left(\mathbf{F}_{\mathbf{f}}=\mathbf{C}_{\mathbf{f}}=0\right)$ or measurement noise. The simulated system includes three coils with 930 turns placed at $2 \pi / 3 \mathrm{rad}$ on a circle of $75 \mathrm{~mm}$ radius, and a capsule which mass is $10 \mathrm{~g}$.

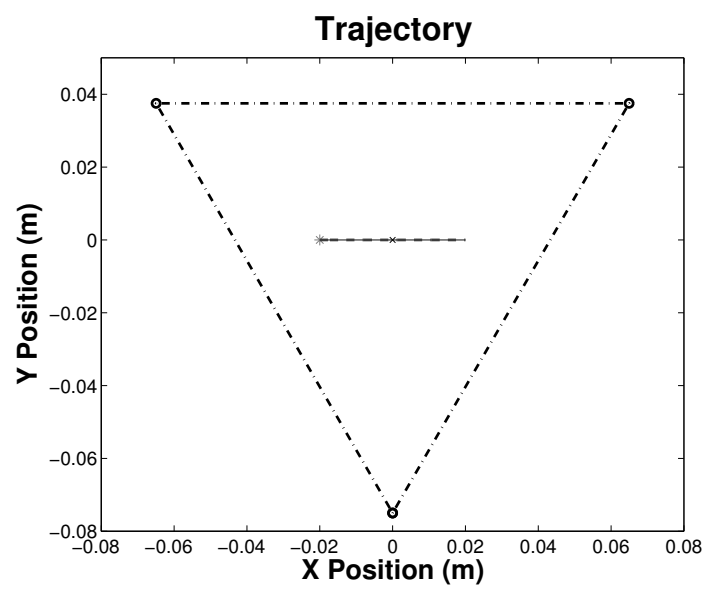

Fig. 5: Position followed by the capsule (plain). The desired trajectory (dashed) is a straight line, the $*$ is the starting point, and the magnetization is in the same direction as the trajectory.

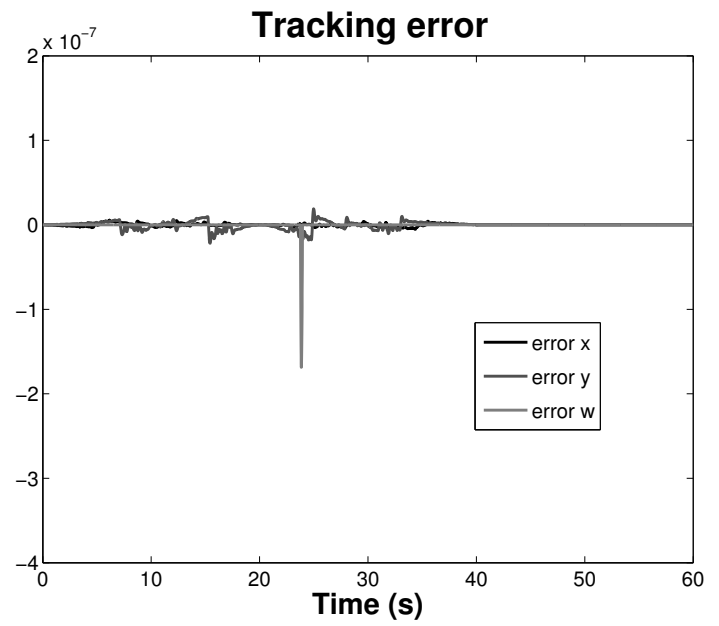

Fig. 6: Tracking error during the trajectory Fig. 5.

On Fig. 5 the desired trajectory $\mathbf{P}^{*}$, a $40 \mathrm{~mm}$ long straight line, and the realized trajectory are displayed. They match very accuratly, with a maximal error of $2.19 \cdot 10^{-5} \mathrm{~mm}$ in position and $1.69 \cdot 10^{-7} \mathrm{rad}$ in orientation. This correspond to the numerical round-off errors.

Moreover, the currents flowing through the coils to realize this trajectory are shown on Fig. 7 and do not exceed 0.35 A. Consequently, we can conclude that the trajectory is physically admissible.

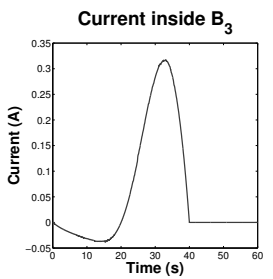

(a)

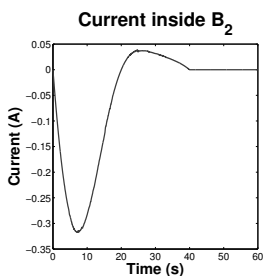

(b)

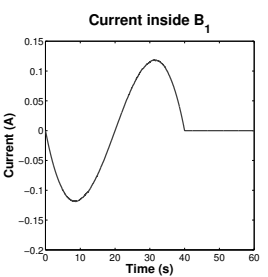

(c)
Fig. 7: Currents inside the coils during the trajectory Fig. 5.

Now, let us observe the angles between the columns of $\left[A_{F}\right]$ along this trajectory. Fig. 8 shows that, as expected, during the whole trajectory, there is always at least one of the angles which is close to $\pi / 2 \mathrm{rad}$. This means that the algorithm is always able to construct a base for the forces in the horizontal plane, and thereby to find a linear combination that gives the desired effort. Moreover, the simulation did not encounter any loss of rank of the matrix $[A]$, which is also normal for a properly planned trajectory. However the two angles come close to $\pi$, meaning that two columns align.

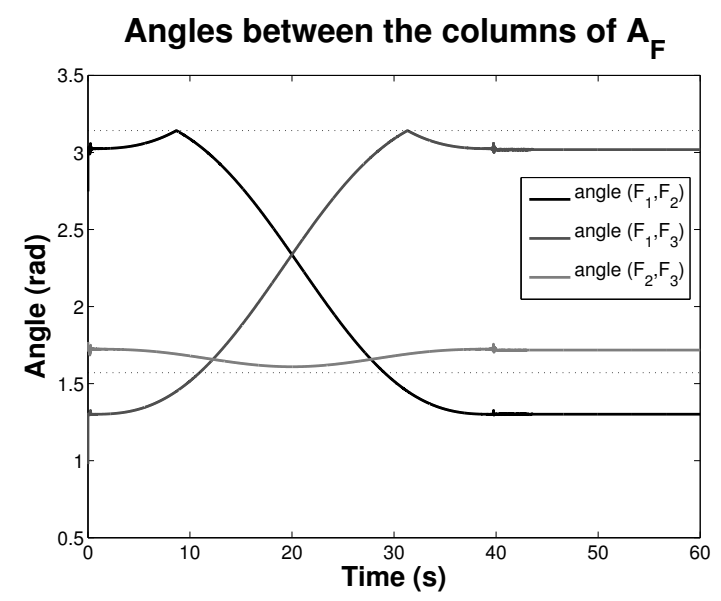

Fig. 8: Angles between the direction of the force created by each coil during the trajectory Fig. 5.

However, executing the simulation for different trajectories led us to find that some of them induce a loss of control. This is illustrated on Fig. 9a and 9b, where the desired trajectory is the same trajectory as the first one, but this time with the capsule orientation perpendicular to the direction of the trajectory. Notice that the control is lost on the orientation. Furthermore, the currents applied to the coils become very high where the control is lost (Fig. 10) which means that this trajectory is not admissible.

The trajectory was realized with several values of $\varepsilon$, the detection threshold of the rank. Note that in the first case 


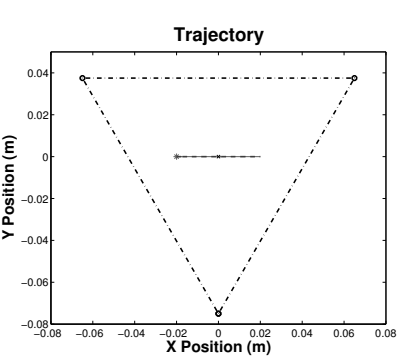

(a)

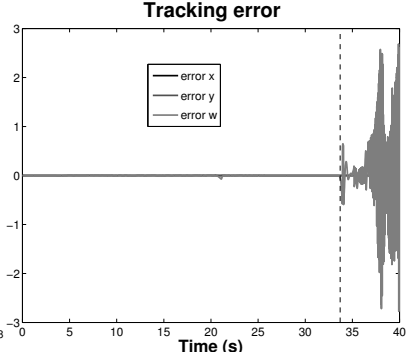

(b)
Fig. 9: (a) Position followed by the capsule (plain). The desired trajectory (dashed) is a straight line, the $*$ is the starting point, and the magnetization is perpendicular to the trajectory. (b) Tracking error during the trajectory.

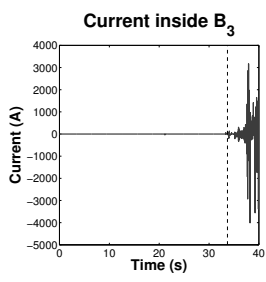

(a)

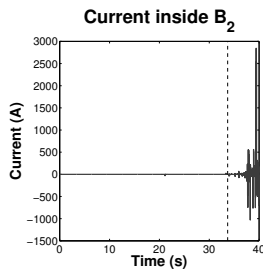

(b)

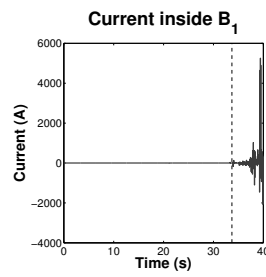

(c)
Fig. 10: Currents inside the coils during the trajectory Fig. 9a.

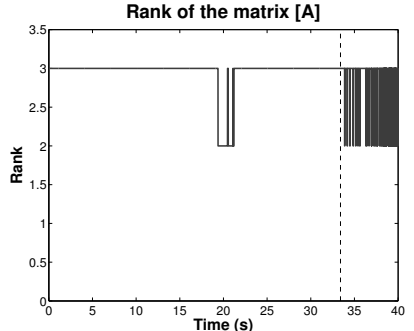

(a) Threshold = 1e-3

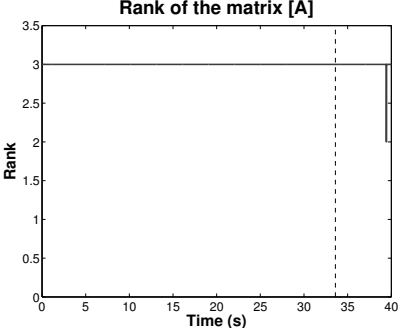

(b) Threshold = 1e-6
Fig. 11: Rank of the matrix [A] during the trajectory Fig. 9a.

( $\varepsilon=10^{-3}$ Fig. 11a) the loss of rank of the manipulation matrix $[A]$ happens before any loss of control, and in the second case $\left(\varepsilon=10^{-6}\right.$ Fig. 11b) the loss of rank is not detected until the control is lost. This highlights that the rank is not a sufficient criteria.

On the contrary, Fig. 12a shows a sudden decrease of the angles between the direction of the forces from each coil. At $t=33.36 \mathrm{~s}$, the starting time of the control loss, the three angles are equal to 0 (Fig. 12b).

In such a case, the three coils can only create a force along the same direction, which actually is orthogonal to the desired trajectory. This fully explains why the current dramatically increase (see Fig. 10 and Fig. 13), yielding unstabilities. To avoid these unstabilities one can define a margin on the angles related to the maximum current admissible by the system (see Fig. 13).

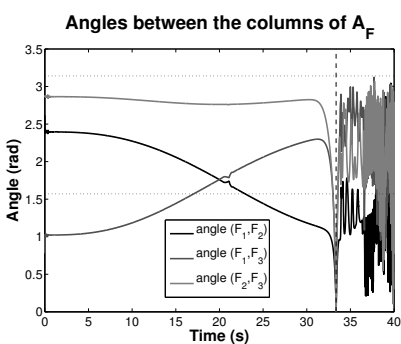

(a)

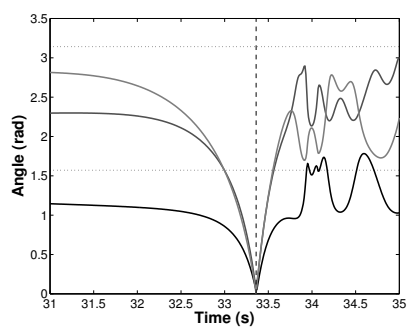

(b)
Fig. 12: (a) Angles between the direction of the force created by each coil during the trajectory Fig. 9a. (b) Zoom in.

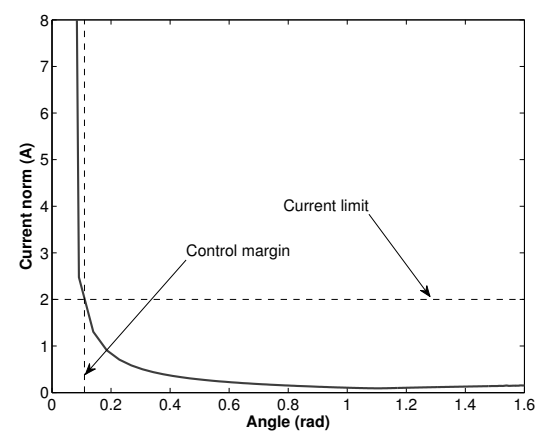

Fig. 13: Norm $\left(\|\mathbf{I}\|_{2}\right)$ of the currents in coils 1 and 2, as a function of the angle $\alpha_{1,2}$ between these coils, during the trajectory Fig. 9a

\section{Discussion}

Conventional devices for magnetic manipulation found in the literature (as [18]) are most of the time composed of four fixed coils, which are placed in a configuration similar to the Helmholtz one (face to face in pairs). A simulation of such a

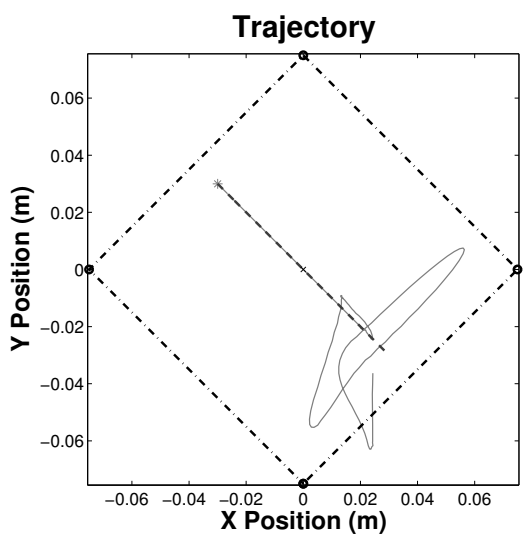

Fig. 14: Simulation output for a 4 fixed coils system.

system was done and the analysis of the angles $\alpha_{i, j}$ between the columns of the matrix $\left[A_{F}\right]$ (whose dimension is $2 \times 4$ in this case) was conducted on this system.

We see that trajectories with singularities on these angles are unusual for this kind of system. Most of the time, the singularities encountered are due to a loss of rank, and the 
perturbation ensuing such a singularity may not induce a strong loss of control. Yet, having found this non-admissible trajectory confirms the need of a proper singularity analysis in magnetic manipulation, whatever the configuration.

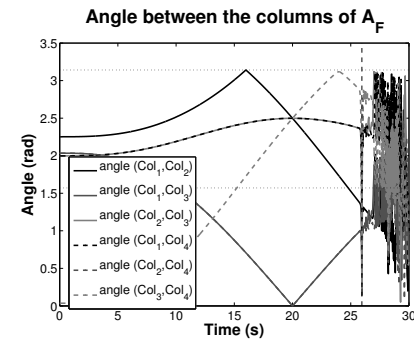

(a)

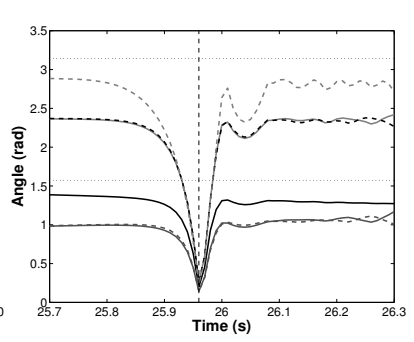

(b)
Fig. 15: (a) Angles between the columns of the matrix $\left[A_{F}\right]$ during the trajectory Fig. 14. (b) Zoom in.

A non-admissible trajectory is displayed Fig. 14. Similarly to the previous simulation, a sudden decrease happens on the angles $\alpha_{i, j}$ (Fig. 15b) at the moment of the control loss $(t=25.96 \mathrm{~s})$. This highlights the relevance of this analysis, and attests that it can be applied to different kind of systems (with mobile or fixed coils).

\section{CONCLUSIONS AND PERSPECTIVES}

This paper studies the manipulability for manipulation systems based on multiple mobile coils. A geometrical analysis allows to check if planned paths are acceptable or not.

This geometrical analysis comes in addition to a more classic numerical criterion (the condition number of the matrix), which is a necessary but insufficient condition. The geometrical criterion easily defines a margin on the angles to ensure the controllability of the system. These results have also been confirmed for magnetic manipulation systems with Helmholtz-like fixed coils.

This study on the angles $\alpha_{i, j}$ is a first interesting approach. However, it is not enough; one must also ensure that the norm of each column is not too low. Indeed, as a column represents the wrench per current unit, a low norm would imply high currents in the coils. A more complete study is being led in order to determine a manipulability criterion adapted to multi mobile coils systems.

Finally, this study reveals that magnetic actuated devices are subject to constraints that may be non-holonomic constraints. One perspective of this work is then the trajectory planning for magnetic manipulation systems.

\section{ACKNOWLEDGMENT}

The authors would like to thank Pierre Renaud, Xavier Weber and Lucas Perdrix for the design of the robotic system seen in Fig. 1. This work was partly supported by the French ANR Labex ACTION ("ANR-11-LABX-01-01").

\section{REFERENCES}

[1] M. Yu, "M2a (tm) capsule endoscopy: A breakthrough diagnostic tool for small intestine imaging," Gastroenterology Nursing, vol. 25(1), pp. 24-27, 2002.

[2] O. Alonso, L. Freixas, J. Canals, E. Susilo, and A. Diguez, "Control electronics integration toward endoscopic capsule robot performing legged locomotion and illumination." in IEEE/RSJ International Conference on Intelligent Robots and Systems, Taipei, Taiwan, October 18-22 2010

[3] J. F. Schenck, C. L. Dumoulin, R. W. Redington, H. Y. Kressel, R. T. Elliott, and I. L. McDougall, "Human exposure to 4.0 tesla magnetic fields in a whole-body scanner," Medical Physics, The International Journal of Medical Physics Research and Practice, vol. 19(4), pp. 1089-1099, 1992.

[4] K. B. Yessin, K. Vollmers, and B. J. Nelson, "Modeling and control of untethered biomicrorobots in a fluidic environment using electromagnetic fields," The International of Robotics Research, vol. 25, p. 527, 2006.

[5] M. P. Kummer, J. J. Abbott, B. E. Kratochvil, R. Borer, A. Sengul, and B. J. Nelson, "Octomag: An electromagnetic system for 5-dof wireless micromanipulation," IEEE Trans. on robotics, vol. 26(6), pp. 1006-1017, 2010.

[6] X. Wang, "Study on magnetic localization and actuation of active capsule endoscope," Ph.D. dissertation, The Chinese University of Hong Kong, August 2006.

[7] O. Felfoul, E. Aboussouan, A. Chanu, and S. Martel, "Real-time positioning and tracking technique for endovascular untethered microrobots propelled by mri gradients," in IEEE International Conference on Robotics and Automation, 2009.

[8] S. Martel, J.-B. Mathieu, O. Felfoul, A. Chanu, E. Aboussouan, S. Tamaz, P. Pouponneau, L. Yahia, G. Beaudoin, G. Soulez, and M. Mankiewicz, "Automatic navigation of an untethered device in the artery of a living animal using a conventional clinical magnetic resonance imaging system," in Applied physics letters, 2007.

[9] S. Tognarelli, V. Castelli, G. Ciuti, C. Di Natali, E. Sinibaldi, P. Dario, and A. Menciassi, "Magnetic propulsion and ultrasound tracking of endovascular devices," Journal of Robotic Surgery, vol. 1, pp. 1-8, 2012.

[10] G. Ciuti, P. Valdastri, A. Menciassi, and P. Dario, "Robotic magnetic steering and locomotion of capsule endoscope for diagnostic and surgical endolumial procedures," Robotica, vol. 28, pp. 199-207, 2010.

[11] A. Mahoney and J. Abbott, "Control of untethered magnetically actuated tools with localization uncertainty using a rotating permanent magnet," in Biomedical Robotics and Biomechatronics (BioRob), 2012 4th IEEE RAS \& EMBS International Conference on. IEEE, 2012, pp. 1632-1637.

[12] G. Lien, C. Liu, J. Jiang, C. Chuang, and M. Teng, "Magnetic control system targeted for capsule endoscopic operations in the stomachdesign, fabrication, and in vitro and ex vivo evaluations," Biomedical Engineering, IEEE Transactions on, vol. 59, no. 7, pp. 2068-2079, 2012.

[13] B. Véron, A. Hubert, J. Abadie, N. Andreff, and P. Renaud, "Advocacy for multi mobile coil magnetic manipulation in active digestive endoscopy." in Workshop on Magnetically Actuated Multiscale Medical Robots, International Conference on Intelligent Robots and Systems., 2012.

[14] B. Véron, J. Abadie, A. Hubert, and N. Andreff, "Magnetic manipulation with several mobile coils towards gastrointestinal capsular endoscopy," in New Trends in Mechanism and Machine Science. Springer, 2013, pp. 681-689.

[15] J. D. Jackson, Classical electrodynamics. John Wiley \& Sons, 1999.

[16] J. Merlet, "Jacobian, manipulability, condition number and accuracy of parallel robots," Robotics Research, vol. 28, pp. 175-184, 2007.

[17] E. Marchand, F. Spindler, and F. Chaumette, "Visp for visual servoing: a generic software platform with a wide class of robot control skills," IEEE Robotics \& Automation Magazine, vol. 12 (4), pp. 40-52, December 2005.

[18] I. A. Ivan, G. Hwang, J. Agnus, M. Rakotondrabe, N. Chaillet, and S. Régnier, "First experiments on magpier: a planar wireless magnetic and piezoelectric microrobot," in Robotics and Automation (ICRA), 2011 IEEE International Conference on. IEEE, 2011, pp. 102-108. 